\title{
Probabilistic Placement of Wind Turbines in Distribution Networks
}

\author{
Tohid Sattarpour', Mohammad Sheikhi², Sajjad Golshannavaz1, Daryoush Nazarpour ${ }^{1}$ \\ 'Department of Electrical Engineering, Urmia University, Urmia, Iran \\ 2Department of Electrical and Computer Engineering, Gazi-Tabatabayi Engineering Faculty, West
}

Cite this article as: T. Sattarpour, M. Sheikhi, S. Golshannavaz, D. Nazarpour, "Probabilistic Placement of Wind Turbines in Distribution Networks", Electrica, vol. 18, no: 2, pp. 234-241, 2018.

\begin{abstract}
This study presents an efficient approach for determining the optimal locations of wind turbines (WTs) in distribution systems, which considers the existing uncertainties in the power generation of WTs and the load demand of consumers. The daily load profiles of the seasonal and geographical-dependent behaviors of WTs are also considered. The proposed probabilistic approach is based on scenario tree modeling, and each scenario is assessed in regard to power loss minimization. Then, the TOPSIS (technique for order preference by similarity to an ideal solution) method is adopted to regulate the optimal placement of WTs considering the average value and the standard deviation of active power losses as possible attributes. This approach enables a multi-attribute analysis of the search space to yield a more efficient solution. Detailed simulation studies, conducted on IEEE 33-bus test system, are utilized to examine the effectiveness of the proposed method. The results of this study are discussed in depth.
\end{abstract}

Keywords: Wind turbine, uncertainty, scenario tree modeling, optimal placement

\section{Corresponding Author: \\ Tohid Sattarpour}

E-mail:

t.sattarpour@urmia.ac.ir

Received: 27.01 .2018

Accepted: 25.04 .2018

(c) Copyright 2018 by Electrica

Available online at

http://electrica.istanbul.edu.tr

DOI: 10.26650/electrica.2018.001

\section{Introduction}

Ensuring the secure planning of power systems has become an important and critical matter in recent years, along with the development of smart and complex systems [1]. Distributed generations (DGs) are new technologies supporting the evolution of smart distribution grids. These units sensibly contribute to increased system reliability and enhanced power quality metrics [2]. The most general kinds of DGs are the renewable-based and conventional diesel-based units [3]. As societies are faced with environmental and economic hurdles ahead of soaring energy demands, deployment of green energy technology is now at the center of attention. Furthermore, significant technical problems such as improving the voltage profile and minimizing the power loss are contemplated as DG-driven technical achievements.

The role of wind turbines (WTs) in minimizing power losses and improving the voltage profile has been carefully assessed in the literature [4]. The main concern in regard of green energy technologies, such as WTs, has to do with their intermittent power generation. To avert such technical flaws, distribution network operators (DNOs) need to establish efficient tools to investigate existing uncertainties. There are different approaches for accommodating the uncertainties of distribution networks. The Monte Carlo simulation (MCS) technique, although portraying a high-resolution and precise manner, is a high computational approach [5]. Scenario tree modeling is one of the best techniques to include the impact of uncertainties. This approach reduces the computational burden of the analyses and maintains an adequate accuracy of the computation procedure [6]. 
Several studies have focused on optimizing the impacts of renewable energies in distribution networks. The authors have presented a probabilistic model of WTs and photovoltaics (PV) comparing their possible impacts [7]. The results, based on this approach, have been compared to that of the symmetric two point estimate method (S2PEM), the Gram-Charlie method, and the Latin hypercube sampling method. Probabilistic operational management of a microgrid was investigated [8]. A self-adaptive gravitational search algorithm was utilized to tackle the optimization procedure. A multi-objective programming method is proposed for reserve and energy planning of intelligent distribution systems [9]. A probabilistic load flow method was devised based on the PEM [3]. In a long-term fashion, analyzed the incorporation of WTs based on a combined MCS method and market-based optimal power flow (OPF) approach [11]. Authors have presented efficient methods for probabilistic calculation of wind energy injections to distribution systems [12]. This aim is pursued based on MCS and particle swarm optimization (PSO) techniques. Although a considerable effort has been dedicated in uncertainty analyses of intermittent wind energy generation and the load profile of the network, their concurrent analyses have not been tailored accurately.

This study aims at establishing an efficient probabilistic approach to determine the optimal location of WTs in distribution systmes. In this manner, the existing uncertainties in both power generation of the WTs and load demand of the consumer are modeled with suitable probability density functions (PDFs). Daily load profile for each season and the geographical-dependent behavior of WTs are taken into account as well. The proposed probabilistic approach deploys scenario tree modeling within which each scenario is investigated in regard of power loss minimization. Afterwards, the technique in order of preference by similarity to ideal solution (TOPSIS) is triggered to regulate the optimal placement strategy based on the average value and the standard deviation of the active power losses. As can be seen, a multi-attribute analysis of the search space is contemplated to yield in a more efficient solution. Detailed simulation studies, conducted on IEEE 33bus test system, are deployed to scrutinize the effectiveness of the proposed approach.

This paper continues as follows. The uncertainties which are involved in the proposed probabilistic approach are introduced in section II. The mathematical skeleton of the proposed placement approach is thoroughly addressed in section III. The evaluation of the model with a case study is described in section IV. Section $V$ eventually concludes the manuscript.

\section{Uncertainity Modeling}

As mentioned earlier, the uncertainties in the load demand profile and the generated power of WTs are considered here. These profiles are extracted on an hourly basis for each of the seasonal periods. Each of these uncertainties is modeled as follows.

\section{Load Demand Uncertainty}

The amount of demand, which is consumed in each hour needs to be forecasted. Generally, it is modeled with a normal PDF [13]. The following representation is considered:

$$
\operatorname{PDF}\left(D_{h}^{s}\right)=\frac{1}{\sqrt{\left(2 \pi\left(\sigma_{h}^{s}\right)^{2}\right)}} \exp \left(-\frac{\left(D_{h}^{s}-\mu_{h}^{s}\right)^{2}}{2\left(\sigma_{h}^{s}\right)^{2}}\right)
$$

Here, $D_{h}^{s}$ is the power demand. Also, $\mu_{h}^{s}$ and $\sigma_{h}^{s}$ represent the mean and standard deviation of demand, respectively. Scenario tree modeling is deployed for the uncertainty handling process. The states number is sensibly designated, as the number of small intervals decreases the modeling accuracy while the number of large intervals increases the computational burden and provokes problem complexity. The mean value of each state is used to compute the variables of output in that specific state. The probability of each interval is designed as follows:

$$
P(D)_{\text {interval }}=\int_{D L_{1}}^{D L_{2}}\left(\frac{1}{\sqrt{\left(2 \pi \sigma^{2}\right)}} \exp \left(-\frac{(D-\mu)^{2}}{2 \sigma^{2}}\right)\right) \times \mathrm{dD}
$$

Where, $D_{L 1}$ and $D_{L 2}$ are respectively, the minimum and maximum bounds of load demand at each interval.

\section{Wind Turbine Modeling}

In this study, the WT intermittent power generation is demonstrated as a Rayleigh PDF. A Rayleigh PDF is a special case of Weibull PDF in which the shape index is equal to 2 . Such an assumption is widely applied in similar studies as a appropriate explanation of wind speed performance [13]. This behavior is represented as follow:

$$
\operatorname{PDF}\left(V_{h}^{s}\right)=\left(\frac{k \times V_{h}^{s}}{\left(C_{h}^{s}\right)^{2}}\right)^{(k-1)} \times \exp \left(-\left(\frac{V_{h}^{s}}{C_{h}^{s}}\right)^{k}\right)
$$

Where, $\mathrm{k}$ is the shape factor which is equal to $2(\mathrm{k}=2) . V_{h}^{s}$ and $C_{h}^{s}$ denote the wind speed forecasted value and its scale factor, respectively. Therefore, the scaling index can be modeled as follows: 


$$
\begin{aligned}
& V_{h_{\text {mean }}}^{s}=\int_{0}^{\infty} V_{h}^{s} \times \operatorname{PDF}\left(V_{h}^{s}\right) \times d V_{h}^{s}= \\
& \int_{0}^{\infty} \frac{2 \times\left(V_{h}^{s}\right)^{2}}{\left(C_{h}^{s}\right)^{2}} \times \exp \left(-\left(\frac{V_{h}^{s}}{C_{h}^{s}}\right)^{2}\right) \times d V_{h}^{s}=\frac{\sqrt{\pi}}{2} \times C_{h}^{s} \\
& C_{h}^{s}=1.128 \times V_{h_{\text {mean }}}^{s}
\end{aligned}
$$

The generated power of a typical WT in each hour is determined based on a WT power curve. This feature is interpreted as follows:

$$
P_{h}^{s}(w)= \begin{cases}0 & v_{\text {mean }} \leq v_{\text {in }}^{c} \\ \frac{v_{\text {mean }}-v_{\text {in }}^{c}}{v_{\text {rated }}-v_{\text {in }}^{c}} \times \mathrm{P}_{r}^{w} & v_{\text {in }}^{c} \leq v_{\text {mean }} \leq v_{\text {rated }} \\ \mathrm{P}_{r}^{w} & v_{\text {rated }} \leq v_{\text {mean }} \leq v_{\text {out }}^{c} \\ 0 & v_{\text {out }}^{c} \leq v_{\text {mean }}\end{cases}
$$

In (6), $\mathrm{P}_{r}^{w}$ speaks to the rated power of WT and $P_{h}^{s}(w)$ is its generated power in hour $h$ and season $s$. As well, $v_{\text {out }}^{c}$ is the cut-out speed, $v_{i n}^{c}$ is the cut-in speed, and $v_{\text {rated }}$ is the rated speed of the WT.

The output power in each interval is achieved by the mean value of each state. The probability of each interval is calculated as follows:

$$
P(w)_{\text {interval }}=\int_{V_{L 1}}^{V_{L 2}}\left(\frac{2 \times V_{\text {mean }}}{C^{2}} \exp \left(-\left(\frac{V_{\text {mean }}}{C}\right)^{2}\right)\right) \times d v_{\text {mean }}
$$

Where, $V_{L 1}$ and $V_{L 2}$ represent the lower and upper bounds of each interval, respectively. Moreover, $C$ stands for the scale factor and $V_{\text {mean }}$ is the mean value of wind speed.

\section{Scenario Tree Formation}

Scenario tree modeling is deployed to define a set of scenarios in the optimal placement of WTs. The combination of load demand and wind speed states end in different scenarios. Each scenario contains two levels of demand value and wind generation accompanied with a particular probability value. The probability of each scenario is calculated based on (8) whose terms are calculated in (2) and (7). As shown in (9), the cumulative summation of all scenarios is equal to one.

$$
\begin{aligned}
& \Pi_{s}=P(\mathrm{D})_{\text {interval }} \times P(w)_{\text {interval }} \\
& \sum_{s=1}^{N_{S}} \Pi_{s}=1
\end{aligned}
$$

Where, $\Pi_{s}$ is the probability of each scenario and $N_{S}$ is the number of scenarios.

\section{The Proposed Methodology}

\section{Objective Functions}

As the proposed approach establishes a TOPSIS-based multi-attribute approach, two objectives are determined as the main attributes of the proposed study. In this context, the first objective function in (10) minimizes the active power losses in distribution feeders and the second one seeks a solution with the minimized variation of the active power losses. It will be assumed that the total power loss of the network is obtained as follow:

$$
\left[P(\text { loss })=\sum_{k=1}^{N_{K}}\left|I_{k}\right|^{2} \times R_{k}\right], \quad k \in \Omega_{B r}
$$

Where, $\Omega_{B r}$ and $K$ denote the set and index of branches, respectively. $I_{k}$ and $R_{k}$ show the current magnitude and resistance of branches, respectively. By determing of the active power losses and probability of each scenario, the expected value (EV) and standard deviation (SD) of different scenarios in each hour are calculated as follows:

$$
\begin{aligned}
& E V=\sum_{s=1}^{N_{S}} \Pi_{s} \times P_{s}(\text { loss }) \\
& S D=\sqrt{\left\{\sum_{s=1}^{N_{S}} \Pi_{s} \times\left(P_{s}(\text { loss })\right)^{2}\right\}-\left\{\left(\sum_{s=1}^{N_{S}} \Pi_{s} \times\left(P_{s}(\text { loss })\right)^{2}\right\}\right.}
\end{aligned}
$$

Here, $P_{s}$ (loss) represents the distribution system power losses in scenario s. Both (11) and (12) are considered as the investigated attributes in the proposed TOPSIS-based probabilistic approach. Accurate forecast of wind power generation or load demand is important for distribution companies. An erroneous estimation ends with an additional energy transfer from the substation transformer, which poses monetary losses. Additionally, this point ends in technical hurdles. Therefore, SD of the power losses is recognized as one of the attribute inaccurate evaluations of the results. The minimum value contribute to a better solution in regard of WT placement.

\section{Constraints}

In each of the scenarios, the nodal power balance should be satisfied. This necessity is denoted based on the constraints represented in (13) and (14). These equations are modified to include the power generation of WTs, load demands, and the transferred power from the main substation.

$$
\begin{aligned}
& P_{g_{i, h, s}}+P_{W T_{i, h, s}}-P_{L_{i, h, s}}=\sum_{j \in \Omega_{i}} P_{i j}\left(V_{i, h, s}, \mathrm{~V}_{j, h, s}, Y_{i j}, \theta_{i j}\right) \\
& Q_{g_{i, h, s}}-Q_{L_{i, h, s}}=\sum_{j \in \Omega_{i}} Q_{i j}\left(V_{i, h, s}, \mathrm{~V}_{j, h, s}, Y_{i j}, \theta_{i j}\right)
\end{aligned}
$$

Where, $P_{W T_{i, h, s}}$ is active power generation of WT. $P_{L_{i, h, s}}$ and 
$Q_{L_{i, h, s}}$ represent the active and reactive power loading of each bus, respectively. $V_{h, s}$ indicates the bus. Finally, $\theta_{i j}$ and $Y_{i j}$ show the phase angle and magnitude of the feeder's admittance, respectively.

In this study, some other constraints, such as the permissible range of voltage magnitude, the rated capacity of substation transformer, and the permissible range of apparent power flow through each distribution feeder, have been considered.

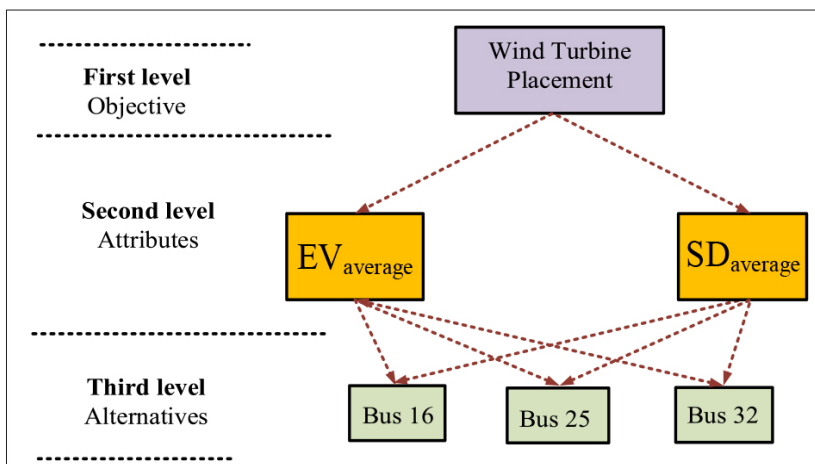

Figure 1. The three-level hierarchy for sorting the possible candidates

\section{TOPSIS Approach}

Prioritizing the candidate buses for the optimal probabilistic placement of WTs is assessed based on the TOPSIS approach. In this manner, a three-level hierarchy is shown in Figure 1. As it can be seen, the objective is located in the first level, attributes are located in the second level, and the third level is considered as alternatives (candidate buses). Regarding the power losses obtained at each scenario and the standard deviation of the results as the attributes, the following steps are conducted.

Step (1): Making a decision matrix based on an entropy technique for three alternatives and two attributes as shown in Table. 1.

In this table, EV and SD are the attributes. Furthermore, the three candidate buses are the alternatives. The average values of EV and SD at each candidate bus can be calculated as follows:

$E V_{\text {average }}=\frac{\sum_{h=1}^{24} E V_{h}}{24}$

Table 1. EV and SD of power losses in spring season

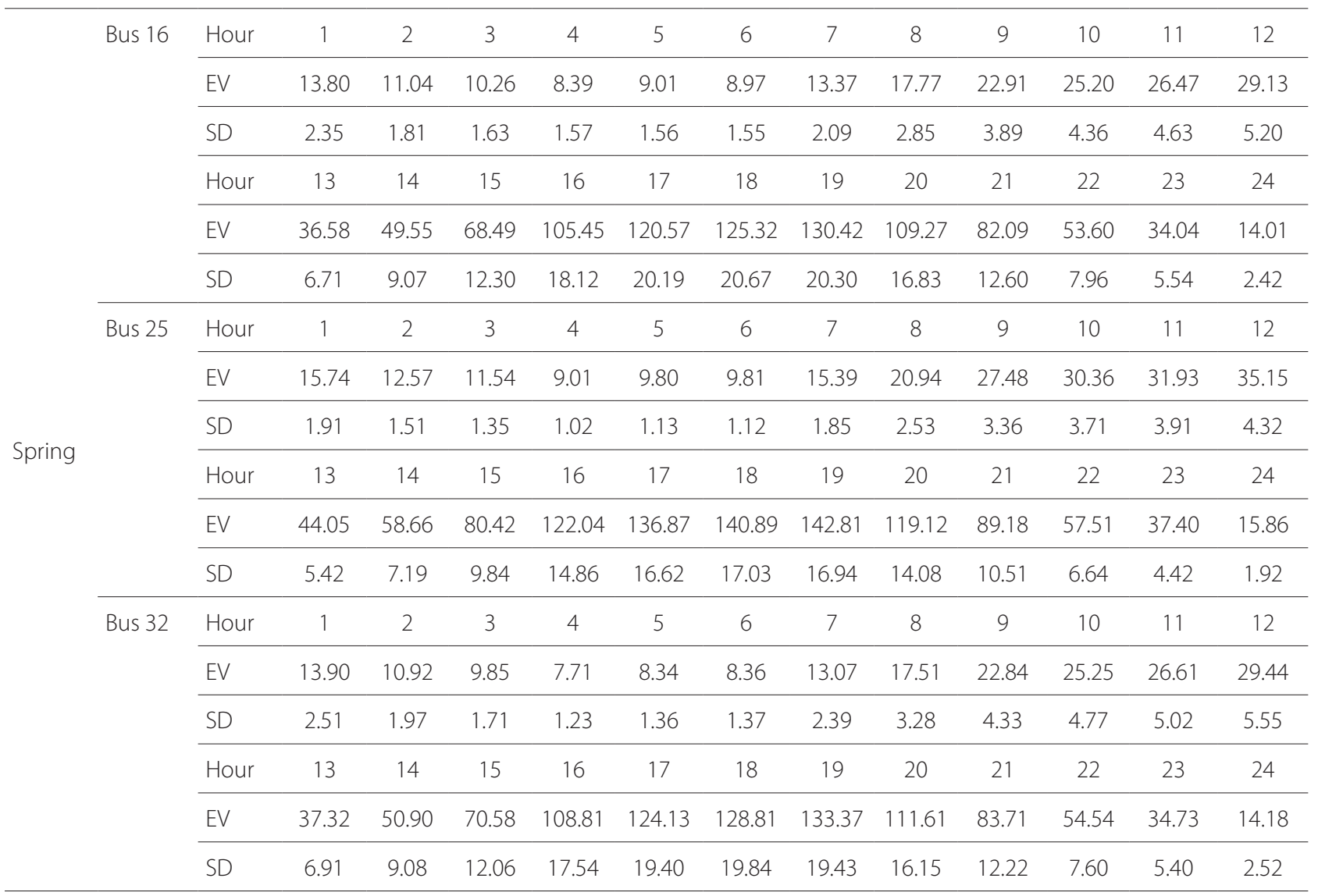


$S D_{\text {average }}=\frac{\sum_{h=1}^{24} S D_{h}}{24}$

Step (2): Decision matrix [A] is normalized based on (17):

$r_{l u}=\frac{a_{l u}}{\sqrt{\sum_{l=1}^{m} a_{l u}^{2}}}$

Where, $a_{l u}$ is a decision matrix element and $m$ is an alternatives quantity.

Step (3): Making the matrix named weighted normalized as $X$.

$X_{l u}=W_{u} \times r_{l u}$

$\sum_{u=1}^{n} W_{u}=1$

It should be noted that each of the two attributes in this study takes a similar weight. Each attribute's weight is considered to be $0.5\left(W_{1}=W_{2}=0.5\right)$.

Step (4): The best and worst answer regarding each attribute are determined in this step. $X_{u}^{+}$as the best answer is measured for the positive and negative criteria as the maximum and minimum values. Also, $X_{u}^{-}$as the worst answer is measured for the positive norm as the minimum value and for the negative norm as the maximum value.

$X_{u}^{+}=\left\{\left(\max X_{h u} \mid u \in u^{+}\right),\left(\min X_{h u} \mid u \in u^{-}\right)\right\} \quad l=1, \ldots, m$

$X_{u}^{-}=\left\{\left(\min X_{l u} \mid u \in u^{+}\right),\left(\max X_{l u} \mid u \in u^{-}\right)\right\} \quad l=1, \ldots, m$

Where, $u$ and I show the $u$-th attribute and I-th alternative, respectively.

Step (5): In this step, the distance of each alternative with the best and worst answers are modeled by $S_{l}^{+}$and $S_{l}^{-}$as follows:

$$
\begin{array}{ll}
S_{l}^{+}=\sqrt{\sum_{u=1}^{2}\left(X_{l u}-X_{u}^{+}\right)^{2}} & l=1, \ldots, m \\
S_{l}^{-}=\sqrt{\sum_{u=1}^{2}\left(X_{l u}-X_{u}^{-}\right)^{2}} & l=1, \ldots, m
\end{array}
$$

Step (6): The mean distance between worst answer and each alternative are modeled as follows:

$$
C_{l}=\frac{S_{l}^{-}}{S_{l}^{-}+S_{l}^{+}} \quad l=1, \ldots, m
$$

Step (7): Sorting the alternatives by considering the values which obtained as $C_{l}$. It should be noted that a higher $C_{l}$ with its higher distance with worst answer is selected as candidate bus (the most effective alternative).

\section{Model of Evaluation on A Case Study}

The proposed probabilistic approach is tested on IEEE 33-bus, depicted in Figure 2. The load point's reactive and active powers and the branches information are taken from [14]. Gathered daily load profiles corresponding to different seasons are shown in Figure 3. Considering the typical distribution system in its basic structure, the total peak demand is equal with 3.715 MW and 2.3 MVA. As it is clearly seen, peak hours are different in different seasons. The mean value of normal PDF is taken equal to the forecasted value. Moreover, the standard deviation of load demand is supposed to be equal to $5 \%$. Bus 1 is supposed to be the substation bus and linked to the sub-transmission grid. Three different candidate buses are nominated as the placement locations of WTs. These buses include 16, 25, and also 32. Since there is a limited budget for placement of

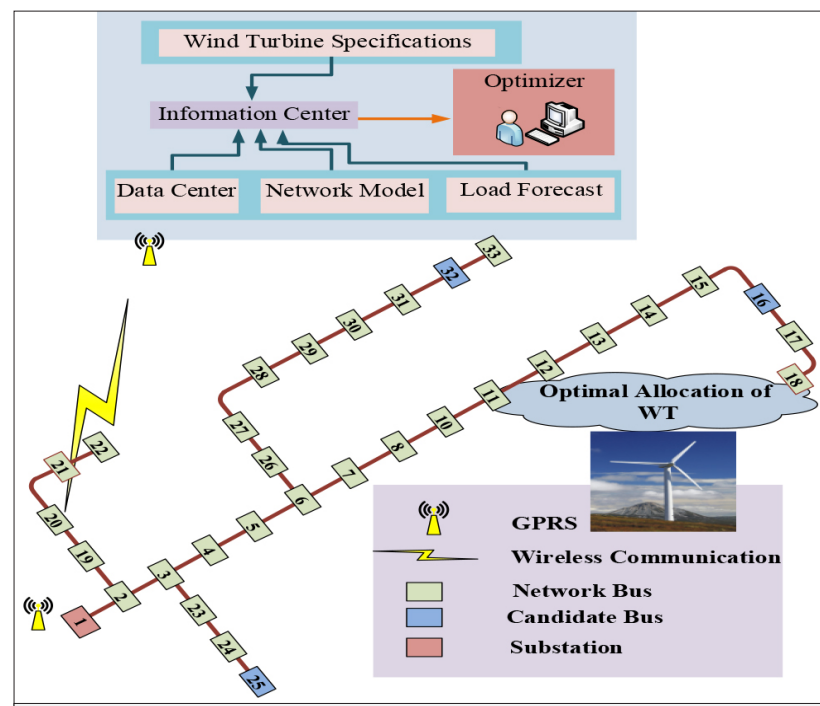

Figure 2. Single line diagram of IEEE-33 bus test system

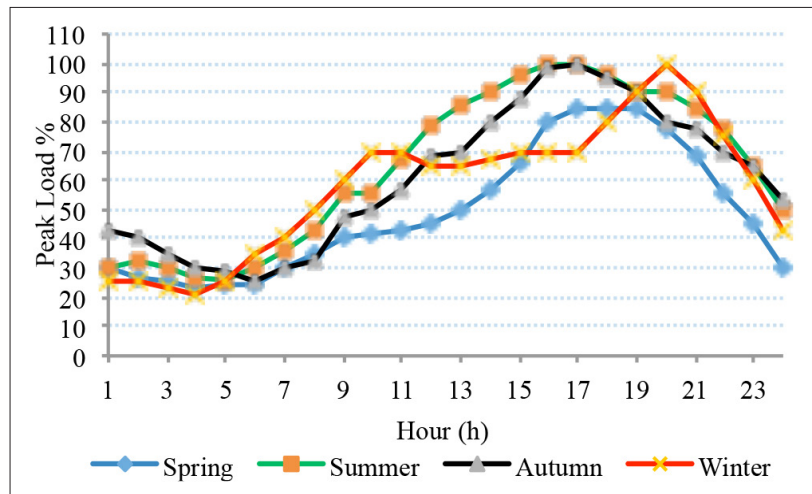

Figure 3. Daily load curves at different seasons 

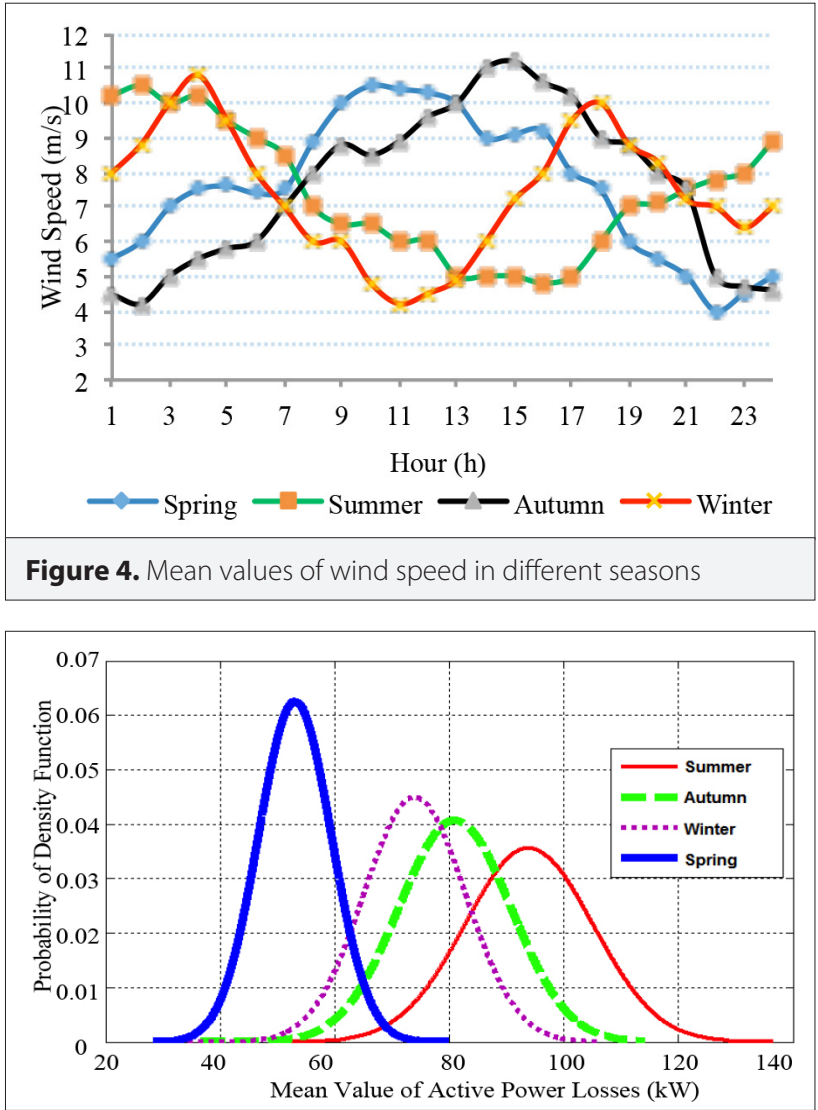

Figure 5. Optimum results for PDF of power losses

Table 2. Attributes and alternatives in WT placement

\begin{tabular}{|c|c|c|c|}
\hline & Bus & $\mathrm{EV}_{\text {average }}$ & $\mathrm{SD}_{\text {average }}$ \\
\hline \multirow[t]{3}{*}{ Spring } & 16 & 46.9073 & 7.7619 \\
\hline & 25 & 53.1108 & 6.3785 \\
\hline & 32 & 47.7805 & 7.6630 \\
\hline \multirow[t]{3}{*}{ Summer } & 16 & 85.4810 & 13.2103 \\
\hline & 25 & 93.9387 & 11.2007 \\
\hline & 32 & 87.0475 & 12.8606 \\
\hline \multirow[t]{3}{*}{ Autumn } & 16 & 70.7984 & 11.8479 \\
\hline & 25 & 81.0212 & 9.8031 \\
\hline & 32 & 72.7659 & 11.5566 \\
\hline \multirow[t]{3}{*}{ Winter } & 16 & 65.9510 & 10.8804 \\
\hline & 25 & 73.9491 & 8.8625 \\
\hline & 32 & 67.3537 & 10.4717 \\
\hline
\end{tabular}

WTs, only one WT is implanted on the network. WTs are operating in unity power factor, i.e., they have not participated in reactive power exchanges. The WT rated capacity is assumed
Table 3. Ranking of the candidate buses for WT placement based on TOPSIS approach

\begin{tabular}{llll}
\hline & \multicolumn{1}{c}{ Bus } & $\boldsymbol{C}_{\boldsymbol{l}}$ & Final Rank \\
\hline \multirow{2}{*}{ Spring } & 16 & 0.3982 & 2 \\
\cline { 2 - 4 } & 25 & 0.6018 & 1 \\
\hline Summer & 16 & 0.3904 & 3 \\
\cline { 2 - 4 } & 25 & 0.3721 & 3 \\
\hline Autumn & 32 & 0.6279 & 1 \\
\cline { 2 - 4 } & 16 & 0.4087 & 2 \\
\hline \multirow{2}{*}{ Winter } & 32 & 0.4241 & 2 \\
\cline { 2 - 4 } & 16 & 0.5759 & 1 \\
\cline { 2 - 4 } & 25 & 0.4207 & 3 \\
\cline { 2 - 4 } & 32 & 0.3671 & 3 \\
\hline
\end{tabular}

as $500 \mathrm{~kW}$. Regarding the power curve specifications, the cut-in speed is equal to $3 \mathrm{~m} / \mathrm{s}$, rated speed is determined as $12 \mathrm{~m} / \mathrm{s}$, and cut-out speed is denoted as $25 \mathrm{~m} / \mathrm{s}$. The average hourly wind speed at each season is shown in Figure 4.

Table 1 shows the EV and SD of power losses attained in different buses and at each hour of a day in spring season. Due to hourly differences in wind speed and load demand, different values of EV and SD are attained. For instance, at hour 16, load demand is at $80 \%$ of peak load and the wind speed's mean value is $9.2 \mathrm{~m} / \mathrm{s}$. Accordingly, the EV and SD of power losses in buses 16,25 , or 32 are attained as $(105.45,18.12),(122.04,14.86)$, and $(108.81,17.54)$, respectively. These differences reflect the impact of WT placement in different buses. As shown in Table 2 , three candidate buses have different results considering the average values of EV and SD. It has been earlier elucidated that the optimal placement solution should portray the minimum $\mathrm{EV}$ as well as the minimum SD.

Based on TOPSIS approach, the priorities of WT placement candidates are determined based on (24). In this way, the results for each season are presented in Table 3. Moreover, the largest distance from the worst answer is considered as the final ranking. Consequently, this bus is designated as the best location for installation. Therefore, bus 25 is selected as the best installation location of WT satisfying the minimum power losses.

Also, Table 4, shows the effect of installed WT on the expected mean power loss at each season. In this table, base plan represents the basic structure of the test case without placing WT. This solution is in line with the minimum power losses in the network and portrays a minimum standard de- 
Table 4. Expected results of the case study

\begin{tabular}{lccc}
\hline & \multicolumn{2}{c}{ Mean Value of Power Losses } & \\
\cline { 2 - 3 } & Base Plan & WT Placement & Loss Reduction \\
\hline Spring & $56.70 \mathrm{~kW}$ & $53.11 \mathrm{~kW}$ & $6.33 \%$ \\
\hline Summer & $98.36 \mathrm{~kW}$ & $93.93 \mathrm{~kW}$ & $4.50 \%$ \\
\hline Autumn & $86.44 \mathrm{~kW}$ & $81.02 \mathrm{~kW}$ & $6.27 \%$ \\
\hline Winter & $78.28 \mathrm{~kW}$ & $73.94 \mathrm{~kW}$ & $5.54 \%$ \\
\hline
\end{tabular}

viation of power losses throughout the investigated hours on a yearly base. In this regard, the PDF of power losses for each season considering the installed WT at bus 25 are depicted in Figure 5.

\section{Conclusion}

A probabilistic approach was devised for WTs optimal placement in distribution systems. In this process, the uncertainties in both load demand and power generation of wind turbines were accommodated through the proposed strategy. Suitable PDFs were constructed for representing the uncertain nature of these variables. Scenario tree modeling was applied for proper segmentation of the PDFs and yielding to a set of scenarios. This approach resulted in a number of scenarios to assess the established approach in a probabilistic manner. It was shown that, each of the scenarios results in different EV and SD of power losses. Thus, the placement location of WT was affected in different seasons and candidate installation buses. Accordingly, the TOPSIS approach was deployed to determine the optimal installation buses of WTs considering the EV and SD values as the decision attributes. It was shown that the three installation candidate buses as the possible alternatives contribute to different trends in the reduction of EV and SD values. The proposed approach allocated the optimal installation buses of WTs based on the largest distance from the worst answer. Consequently, the minimized EV and SD values were granted. These remarks are recognized as impressive factors to be concerned by the DNOs in renewable-based DGs optimal placement in distribution systems.

Peer-review: Externally peer-reviewed.

Acknowledgements: The authors would like to thank the respected editor and reviewers for their valuable time in reading the paper and providing constructive comments.

Conflict of Interest: No conflict of interest was declared by the authors.

Financial Disclosure: The authors declared that this study has received no financial support.

\section{References}

1. M. Mezaache, K. Chikhi, C. Fetha, "UPFC Device: optimal location and parameter setting to reduce losses in electric-power systems using a genetic-algorithm method", Transactions on Electrical and Electronic Materials, vol. 17, no. 1, pp. 1-6, 2016.

2. A. Kumar Bohre, G. Agnihotri, M. Dubey, "Optimal sizing and sitting of DG with load models using soft computing techniques in practical distribution system", IET Generation, Transmission \& Distribution, vol. 10, no. 11, pp. 2606-2621, 2016,

3. H. Kirkici, B. Bernstein, "Energy policies and research/development trends in the USA", Transactions on Electrical and Electronic Materials, vol. 11, no. 5, pp. 197-201, 2010.

4. U. Sultana, A. B. Khairuddin, M.M. Aman, A.S. Mokhtar, N. Zareen, "A review of optimum DG placement based on minimization of power losses and voltage stability enhancement of distribution system", Renewable and Sustainable Energy Reviews, vol. 63 pp. 363-378, 2016.

5. D. Šošic, M. Žarkovic, G. Dobric, "Fuzzy-based Monte Carlo simulation for harmonic load flow in distribution networks", IET Generation, Transmission \& Distribution, vol. 9, no. 3, pp. 267-275, 2015.

6. S. Seguin, S. E. Fleten, P. Cote, A. Pichler, C. Audet, "Stochastic short-term hydropower planning with inflow scenario trees", European Journal of Operational Research, 2016,.

7. A. Soroudi, M. Aien, M. Ehsan", A Probabilistic modeling of photo voltaic modules and wind power generation impact on distribution networks", IEEE System Journal, vol. 6, no. 2, pp. 254-259, 2012.

8. T. Niknam, F. Golestaneh, A. Malekpour, "Probabilistic energy and operation management of a microgrid containing wind/ photovoltaic/ fuel cell generation and energy storage devices based on point estimate method and self-adaptive gravitational search algorithm", Energy, vol. 43, pp. 427-437, 2012.

9. A. Zakariazadeh, S. Jadid, P. Siano, "Economic environmental energy and reserve scheduling of smart distribution systems: A multiobjective mathematical programming approach", Energy Conversion and Management, , vol. 78, pp. 151-164, 2014.

10. G. Mokryani, P. Siano, "Evaluating the integration of wind power into distribution networks by using Monte Carlo simulation", Electrical Power and Energy Systems, vol. 53, pp. 244-255, 2013.

11. P. Siano, G. Mokryani, "Probabilistic assessment of the impact of wind energy integration into distribution networks," IEEE Transactions on Power Systems, vol. 28, no. 4, pp. 4209-4217, 2013.

12. S. Surender Reddy, P. R. Bijwe, A. R. Abhyankar, "Joint energy and spinning reserve market clearing incorporating wind power and load forecast uncertainties", IEEE Systems Journal, vol. 9, no. 1, pp. 152-164, 2015,

13. S. Shojaabadi, S. Abapour, M. Abapour, A. Nahavandi, "Simultaneous planning of plug-in hybrid electric vehicle charging stations and wind power generation in distribution networks considering uncertainties", Renewable Energy, 2016, vol. 99, pp. 237-252.

14. S. Chandramohan, N. Atturulu, R.P. Kumudini Devi, B. Venkatesh, "Operating cost minimization of a radial distribution system in a deregulated electricity market through reconfiguration using NSGA method", Electrical Power and Energy Systems, 2010, vol. 32 , no. 2, pp. 126-132. 
Tohid Sattarpur received the B.Sc. and M.Sc. (Hons.) degrees in electrical engineering from Urmia University, Urmia, Iran, in 2012 and 2014, respectively. Currently, he is a Ph.D. student in electrical engineering in the same university. His research interests are centered in smart grid technologies, optimal operation and planning of active distribution networks, and renewable energies.

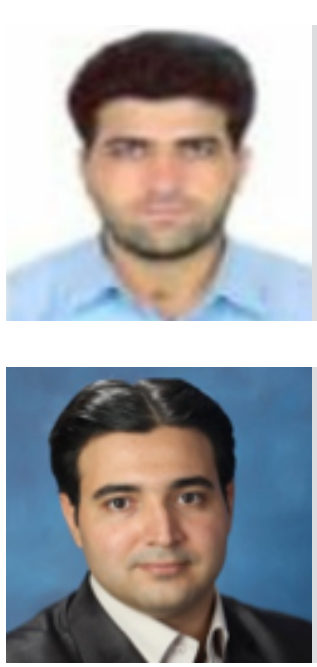

Mohammad Sheikhi received the B.Sc. degree from the Urmia univertsity, Urmia, Iran,. and M.Sc. degrees from Azad university of Tehran, His research interests are in smart distribution grid, renewable energies, and optimal operation of distribution networks.

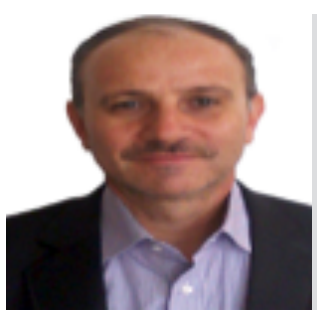

Sajjad Golshannavaz received the B.Sc. (Hons.) and M.Sc. (Hons.) degrees, both in electrical engineering from Urmia University, Urmia, Iran, in 2009 and 2011, respectively. He is currently pursuing the Ph.D. degree at the School of Electrical and Computer Engineering, College of Engineering, University of Tehran, Tehran, Iran. Since 2014 he has been collaborating with the smart electric grid research laboratory, Department of Industrial Engineering, University of Salerno, Salerno, Italy.

Daryoosh Nazarpour received the B.Sc. degree from the Iran University of Science and Technology, Tehran, Iran, in 1982 and the M.Sc. and Ph.D. degrees from University of Tabriz, Tabriz, Iran, in 1988 and 2005, respectively, all in electrical engineering. He is currently an Associate Professor with the School of Electrical and Computer Engineering, Urmia University, Urmia, Iran. His research interests are primarily centered in advanced power electronics and FACTS applications in power systems as well as smart electric grids. 